\title{
Piezoelectric Actuation of Graphene-Coated Polar Structures
}

\author{
Andrei L. Kholkin ${ }^{\circledR}$, Fellow, IEEE, Andrei D. Ushakov, Member, IEEE, Maria A. Chuvakova, \\ Mikhail S. Kosobokov, Andrey R. Akhmatkhanov, Andrei V. Turutin, Maxim V. Chichkov, \\ Ivan I. Kravchenko, Yakov Kopelevich, and Vladimir Ya. Shur ${ }^{(\mathbb{0}}$, Senior Member, IEEE
}

\begin{abstract}
Ferroelectric materials based on lead zirconate titanate (PZT) are widely used as sensors and actuators because of their strong piezoelectric activity. However, their application is limited because of the high processing temperature, brittleness, lack of conformal deposition, and a limited possibility to be integrated with the microelectromechanical systems (MEMS). Recent studies on the piezoelectricity in the 2-D materials have demonstrated their potential in these applications, essentially due to their flexibility and integrability with the MEMS. In this work, we deposited a few layer graphene (FLG) on the amorphous oxidized $\mathrm{Si}_{3} \mathrm{~N}_{4}$ membranes and studied their piezoelectric response by sensitive laser interferometry and rigorous finite-element modeling (FEM) analysis. Modal analysis by FEM and comparison with the experimental results show that the driving force for the
\end{abstract}

Manuscript received December 5, 2019; accepted May 22, 2020. Date of publication June 3, 2020; date of current version September 25, 2020. This work was supported in part by the Russian Foundation for Fundamental Research under Grant 16-29-14050, in part by the Ministry of Education and Science of the Russian Federation in the framework of the Increase Competitiveness Program of MISiS under Grant K2-2019-015, in part by the Project CICECO-Aveiro Institute of Materials financed by national funds through the Portuguese Foundation for Science and Technology/MCTES under Grants UIDB/50011/2020 and UIDP/50011/2020, and in part by the Center for Nanophase Materials Sciences, which is a Department of Energy Office of Science User Facility. The work was also supported by Government of the Russian Federation (Act 211, Agreement 02.A03.21.0006) and by the Ministry of Science and Higher Education of the Russian Federation (state task FEUZ-2020-0054). The equipment of the Ural Center for Shared Use "Modern nanotechnology" UrFU was used. The work of Yakov Kopelevich was supported in part by Conselho Nacional de Desenvolvimento Científico e Tecnológico (CNPq) and in part by the Fundação de Amparo à Pesquisa do Estado de S. Paulo (FAPESP) (Brazil). (Corresponding author: Andrei L. Kholkin.)

Andrei L. Kholkin is with the Department of Physics and CICECOAveiro Institute of Materials, University of Aveiro, 3810-193 Aveiro, Portugal (e-mail: kholkin@ua.pt).

Andrei D. Ushakov, Maria A. Chuvakova, Mikhail S. Kosobokov, Andrey R. Akhmatkhanov, and Vladimir Ya. Shur are with the Institute of Natural Sciences and Mathematics, Ural Federal University, 620000 Ekaterinburg, Russia.

Andrei V. Turutin is with the Laboratory of Functional Low-Dimensional Structures, National University of Science and Technology MISiS, 119049 Moscow, Russia, and also with the Department of Physics and I3N, University of Aveiro, 3810-193 Aveiro, Portugal.

Maxim V. Chichkov is with the Laboratory of Functional LowDimensional Structures, National University of Science and Technology MISiS, 119049 Moscow, Russia.

Ivan I. Kravchenko is with the Center for Nanophase Materials Sciences, Oak Ridge National Laboratory, Oak Ridge, TN 37831 USA.

Yakov Kopelevich is with the Instituto de Física Gleb Wataghin, Universidade Estadual de Campinas, UNICAMP, Campinas 13083-859, Brazil.

This article has supplementary downloadable material available at http://ieeexplore.ieee.org, provided by the authors.

Digital Object Identifier 10.1109/TUFFC.2020.2998976 piezoelectric-like response can be a polar interface layer formed between the residual oxygen in $\mathrm{Si}_{3} \mathrm{~N}_{4}$ and the FLG. The response was about $14 \mathrm{~nm} / \mathrm{V}$ at resonance and could be further enhanced by adjusting the geometry of the device. These phenomena are fully consistent with the earlier piezoresponse force microscopy (PFM) observations of the piezoelectricity of the graphene on $\mathrm{SiO}_{2}$ and open up an avenue for using graphene-coated structures in the MEMS.

Index Terms-Finite-element analysis, graphene, membrane, piezoelectricity, resonance.

\section{INTRODUCTION}

OURRENTLY, inorganic ferroelectrics such as lead zirconate titanate (PZT), lithium niobate (LN), and barium titanate (BT) are widely used as efficient piezoelectrics, pyroelectrics, memory cells, and electrooptic modulators [1]. They possess high switchable polarization, strong piezoelectric response, and remarkable pyroelectric and electrooptic properties, but have a number of disadvantages that preclude their use [2], [3]. First, they are not biologically compatible and require encapsulation for contact with biological environments. Second, their processing is at high temperatures, so their miniaturization and integration with the microelectromechanical systems (MEMS) are difficult. Recent discovery of piezoelectricity in 2-D materials [4], [5] opens up new opportunities for stretchable electronics [6], MEMS, and other electronic components based on the direct and converse piezoelectric effects. Being a 2-D monoatomic material with many unique properties [7], graphene is one of the favorable candidates for these applications. It exhibits a variety of emergent properties such as high thermal conductivity, superior mechanical strength, and extremely high flexibility. Although pristine graphene does not possess any piezoelectric activity due to its intrinsically centrosymmetric crystal structure, polar properties (including piezoelectricity) can be induced by breaking of the inversion symmetry by the adsorption of foreign atoms, by introducing specific in-plane defects, or by nonuniform deformation of graphene layers, in which strain gradients create internal polarization in a material [8]-[11]. Recent studies [12] revealed a strong piezoelectric activity of the graphene flakes deposited on the $\mathrm{Si} / \mathrm{SiO}_{2}$ grating substrates. The piezoelectric properties were evaluated by piezoresponse force microscopy (PFM), which is based on the detection of local deformation caused by the small ac bias applied to the conducting tip of the PFM cantilever. The piezoelectric activity in the 
graphene layers was attributed to the chemical interaction of the graphene atoms with the underlying oxygen from the $\mathrm{SiO}_{2}$ substrate [12]. The local piezoelectric coefficient was sufficiently high, comparable with the values for the best piezoelectric ceramics, e.g., PZT or $\mathrm{BaTiO}_{3}$. However, no macroscopic observation of piezoelectricity has been reported until now due to the extremely thin polar layer preventing the measurements of both direct and converse piezoelectric effects.

In this work, we study piezoelectric resonances in thin silicon nitride membranes covered by graphene flakes with the bottom Al electrode. The piezoelectric response was about $14 \mathrm{~nm}$ at $3.7 \mathrm{kHz}$ under $1 \mathrm{~V}$ applied between the graphene and the 100-nm Al layer. These results provide clear prospects for using graphene as an MEMS material; however, membrane parameters have to be further optimized in order to increase the useful displacements and the desired frequency range.

\section{EXPERIMENT}

In this work, low stress (nonstoichiometric) $1-\mu$ m-thick silicon nitride films were deposited onto the $\mathrm{Si}(100)$ wafers by means of low-pressure chemical vapor deposition (LPCVD) from a gas mixture of dichlorosilane (DCS), $\mathrm{SiH}_{2} \mathrm{Cl}_{2}$, and ammonia in a Tystar horizontal furnace. Etch windows were photolithographically delineated on the wafer backsides followed by a plasma etch to remove the $\mathrm{Si}_{3} \mathrm{~N}_{4}$ layer. After that, the $\mathrm{Si}_{3} \mathrm{~N}_{4}$ membranes were manufactured by wet etching of the nonprotected by nitride silicon in hot $30 \% \mathrm{KOH}$. The membrane dimensions were $6.7 \times 6.7 \mathrm{~mm}^{2}$, and the entire surface of the etched area was covered with graphene flakes, as shown schematically in Fig. 1. Several samples were studied by XRD, but no diffraction peaks other than those belonging to the Si substrate were found, indicating that the silicon nitride films were amorphous; the Si crystallites mentioned in literature [13] were not observed. Al electrodes of $100 \mathrm{~nm}$ in thickness were sputtered through a mask on the nonetched side of the wafer (Fig. 1). Energy-dispersive analysis of X-rays (EDAX) was used to determine the oxygen concentration of about 0.72 at. $\%$ in silicon nitride. Graphene was synthesized on a PlanarTech G2 installation using the CVD method on a copper foil. Acetylene was used as a prosecutor; hydrogen was added to the reactor for dilution in the ratio $\mathrm{C}_{2} \mathrm{H}_{2}: \mathrm{H}_{2}=1: 4$. The process temperature was $1040{ }^{\circ} \mathrm{C}$ and the pressure was 6 Torr. The transfer was made using a film of polymethyl-methacrylate (PMMA), as an intermediate substrate. First, a liquid polymer (4\% solution of PMMA in anisole) was applied to a copper foil with graphene by centrifugation at a speed of $1500 \mathrm{rpm}$ and was baked in an oven at a temperature of $150{ }^{\circ} \mathrm{C}$. Furthermore, copper was etched away in an aqueous solution of chlorinated iron. Then, the obtained PMMA film was washed twice in deionized water and laid on a substrate. After drying in a centrifuge at a speed of $3000 \mathrm{rpm}$, the film was heated on a hot plate at a temperature of $120{ }^{\circ} \mathrm{C}$ to eliminate the wrinkles. After that, the PMMA layer was removed in acetone. The quality of graphene was checked by Raman scattering (Fig. S1), and it was shown that it is indeed FLG. The measurements of electric field displacements were done using the sensitive

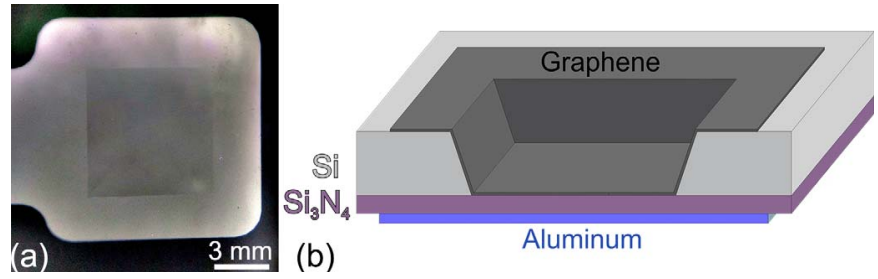

Fig. 1. (a) Optical image of the graphene-coated $6.7 \times 6.7 \mathrm{~mm}^{2} \mathrm{Si}_{3} \mathrm{~N}_{4}$ membrane (bottom view). (b) Schematic of the membrane (side view).

double-beam laser interferometer [14], [15] with the sensitivity of $2 \cdot 10^{-2} \AA$. The beam of the interferometer was positioned in different places of the membrane. Finite-element modeling (FEM) of the membranes was performed using the COMSOL Multiphysics package (version 5.4).

\section{Results And Discussion}

As the studied silicon nitride structure is amorphous, no piezoelectric response was expected from the graphenecoated membranes, except electrostriction measured at the second harmonic of the applied ac bias. Contrary to these expectations, a strong response was found at the first harmonic, as shown in Fig. 2. The results clearly demonstrate that, opposite to common knowledge, graphene-coated membranes exhibit a strong piezoelectric activity more than 30 times exceeding that of electrostriction. The piezoelectric signal was linear with the applied bias [Fig. 2(b)], while the signal at the second harmonic demonstrated a pronounced quadratic behavior with the field [Fig. 2(d)]. The calculated effective electrostriction coefficient measured in the center of a membrane was about $\left(\mathrm{M}_{11}\right)_{\text {eff }} \sim 4.4 \cdot 10^{-13} \mathrm{~m}^{2} / \mathrm{V}^{2}$. This value is much greater than that observed in a similar compound $\mathrm{SiO}_{2}$ $\left(\mathrm{M}_{11} \sim 10^{-15} \mathrm{~m}^{2} / \mathrm{V}^{2}\right)$ [16]. A part of this enhancement could be due to the flexure of the membrane that presents a bimorph structure with the graphene/ $/ \mathrm{Si}_{3} \mathrm{~N}_{4}$ interface as an active layer clamped by the underlying $\mathrm{Si}_{3} \mathrm{~N}_{4}$ and $\mathrm{Al}$ electrode.

In addition, in contrast to piezoelectric quartz $\left(\mathrm{d}_{11}=2.3 \mathrm{pm} / \mathrm{V}\right)$, the effective piezoelectric coefficient of the graphene-coated membrane is about $1 \mathrm{~nm} / \mathrm{V}$ at low frequencies and reached $14 \mathrm{~nm} / \mathrm{V}$ at the fundamental resonance (Fig. S5). This value is comparable with that necessary for the application of membranes as micropumps for drug delivery and other microfluidic devices. For example, poly(vinylidene fluoride-co-trifluoroethylene) (PVDF-TrFE)-based membranes demonstrate the voltageinduced piezoelectric response of about $20 \mathrm{~nm} / \mathrm{V}$ [17]. The advantage of graphene compared with PVDF-TrFE is the ease of deposition, no necessity to pole by stretching, and much greater flexibility that will facilitate the fabrication of ultrathin silicon-based membranes with large displacements.

Membranes are always in tension and, in a rough approximation, their fundamental resonance frequency (for the case of a thin square membrane clamped at the edges) can be written as [18]

$$
f=\frac{\sqrt{2}}{2 L} \sqrt{\frac{T}{\rho t}}
$$



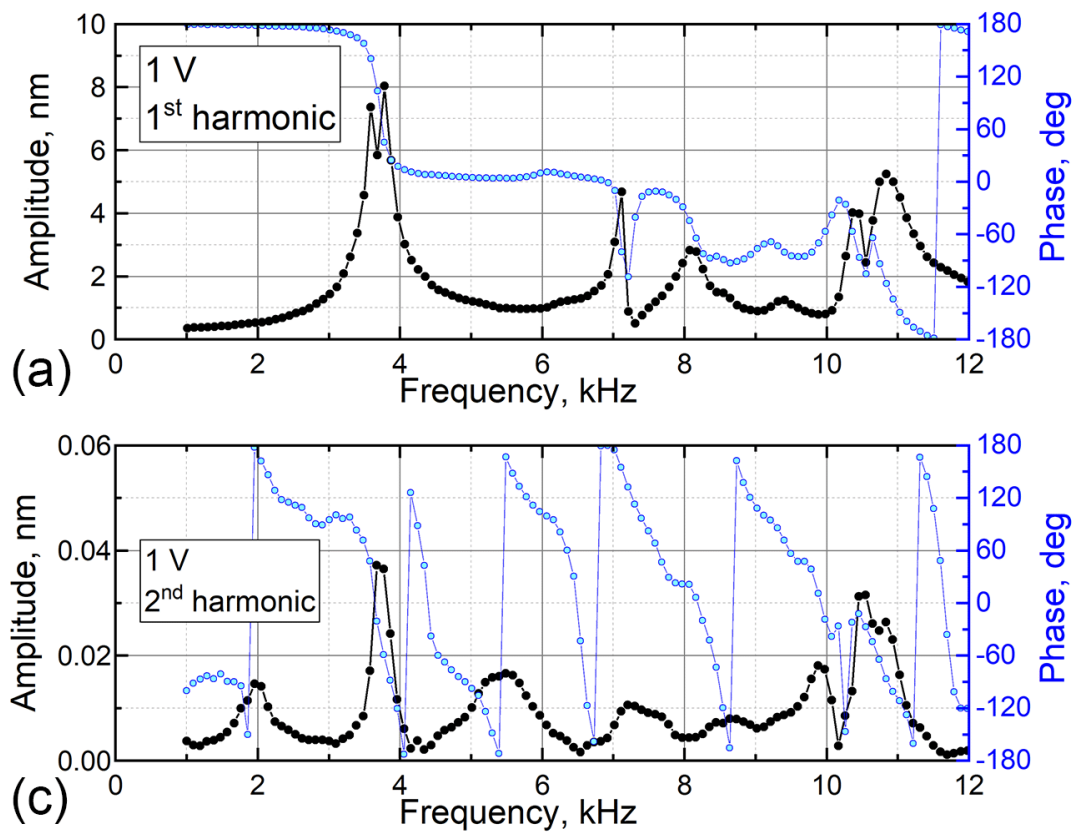

(b)
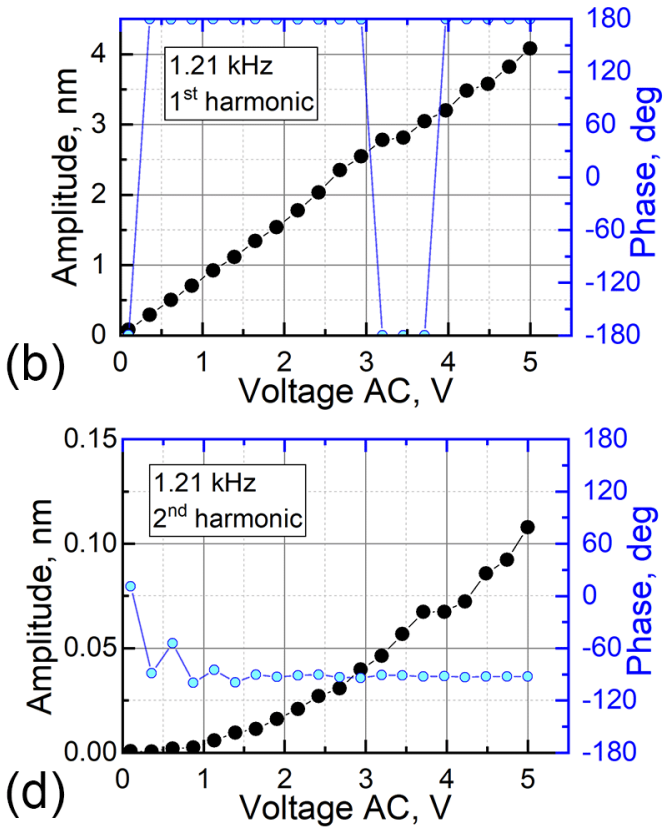

Fig. 2. Frequency dependences of (a) first and (c) second harmonics of the displacement measured in the center of a membrane under an ac voltage of $1 \mathrm{~V}$ between the graphene and Al electrodes. AC voltage dependences of the displacements measured under quasi-static conditions at (b) first and (d) second harmonics.

where $L$ is the side length of the membrane, and $T, \rho$, and $t$ are the tension, density, and thickness of the membrane, respectively. Using this equation, we could estimate the tensile stress to be about 4.7 MPa for our membranes. This sufficiently low stress value could be due to a competition of the compressive stress imposed by the thermal mismatch between the deposited layer and the substrate (introduced on cooling from the deposition temperature) and a larger intrinsic tensile stress, caused by the growth process itself [19], [20]. However, for the rigorous analysis of the piezoelectric displacements induced by graphene, a more quantitative method of FEM is needed as follows.

All the calculations in this work were made by the FEM using a COMSOL Multiphysics software. In the simulation procedure, free (clamped only at the edges) square membrane (with $\mathrm{Al}$ electrode) and square membrane with the fixed part (due to the silver-paste contact between the graphene and the contact wire) have been modeled for extracting the natural frequencies and mode shapes during the graphene-excited vibrations (Fig. S4). The dimensions of the membranes have been taken from the experiment. Only two membrane layers, $\mathrm{Si}_{3} \mathrm{~N}_{4}$ layer and aluminum electrode layer, were considered as being significant in membrane mechanics. The contribution from the mechanical properties of the graphene electrode was apparently neglected due to its negligible thickness. To describe the Dirichlet boundary conditions, external edges along the perimeter of the membrane were assumed to be fixed at zero displacement.

For the optimal mesh geometry, a regular quadrilateral prism was used for the elements. The size of the elements does not exceed $150 \mu \mathrm{m}$ in the plane of the membrane, and at the same time, five elements fit in the membrane thickness (Fig. S2). The initial stress of the $\mathrm{Si}_{3} \mathrm{~N}_{4}$ layer in the membrane was a

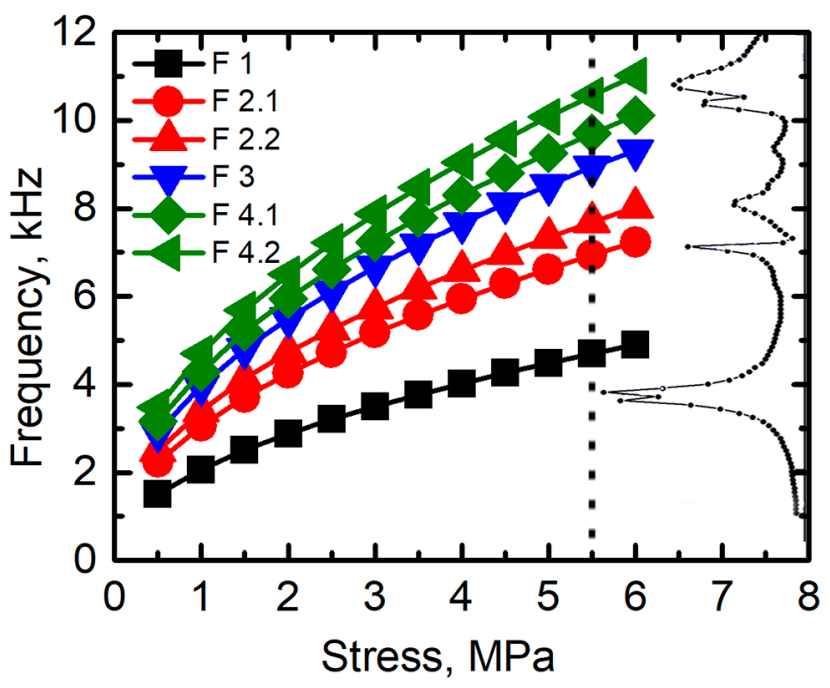

Fig. 3. Calculation results comparing the stress dependence of the piezoelectric resonance of the $\mathrm{Si}_{3} \mathrm{~N}_{4}$ membrane with the clamped electrode (silver paste) with the real resonance spectra. It is seen that the calculated frequencies match the experimental one (right) for the stress of about 5.5 MPa.

parameter in the calculations and varied from 0.5 to $6 \mathrm{MPa}$. The stress was supposed to be distributed uniformly along the membrane plane.

The first calculation set was made for the free membrane [Fig. S4(a)]. Comparison of the calculated stress dependences of the natural frequencies with experimental vibration spectrum did not match all the resonant peaks. Taking into account the fixed part $1.2 \times 1.2 \mathrm{~mm}^{2}$ on the top of the membrane [Fig. S3(a)], which corresponds to the glued electrode used in 
TABLE I

Comparison of the Calculated and Experimentally Measured Resonance Frequencies of Graphene-Coated Membranes

\begin{tabular}{|c|c|c|c|c|c|}
\hline Resonance peak & $\begin{array}{l}\text { Experimental } \\
\text { frequency, } \mathrm{kHz}\end{array}$ & $\begin{array}{c}\text { FEM } \\
\text { frequency } \\
\text { at } 5.5 \mathrm{MPa} \\
\end{array}$ & $\begin{array}{l}\text { Type of } \\
\text { resonance }\end{array}$ & Splitting & $\begin{array}{c}\text { Amplitude, } \\
\text { nm }\end{array}$ \\
\hline $\begin{array}{l}\text { Fundamental } \\
\text { frequency }\end{array}$ & 3.77 & 4.71 & & no & 13.6 \\
\hline $2^{\text {nd }}$ peak & 7.11 & 6.96 & & yes & 7.9 \\
\hline $3^{\text {rd }}$ peak & 8.08 & 7.73 & & yes & 2.46 \\
\hline $4^{\text {th }}$ peak & 9.40 & 8.98 & & yes & 10.9 \\
\hline $5^{\text {th }}$ peak & 10.36 & 9.74 & & no & 4.01 \\
\hline $6^{\text {th }}$ peak & 10.75 & 10.6 & & no & 7.5 \\
\hline
\end{tabular}

the experiment, gave a complete coincidence of the number of peaks and the number of the natural frequencies in the studied frequency range (Table I). Increasing the natural frequency number is due to the membrane symmetry breaking, which leads to the splitting of the second vibration mode (Fig. 2) and the appearance of the new modes at higher frequencies. The peak splitting could be caused by two reasons: 1) the intrinsic elastic anisotropy present in $\mathrm{Si}$ can result in a distribution in the membrane curvature as a function of in-plane angle even if the overlying $\mathrm{Si}_{3} \mathrm{~N}_{4}$ film is isotropic (amorphous) and 2) asymmetric contact on the graphene [Fig. S3(a)] that results in the asymmetric mode shapes as confirmed by FEM modeling [Table I and Fig. S4(b)]. In order to account for the tensile stress on the membranes, we varied the stress in the FEM calculations (Fig. 3). The best match with the experiments was found for the mechanical stress value 5.5 MPa, which is very close to that calculated with (1). A comparison of the experimental values of resonance frequencies with those obtained by FEM is presented in Table I. Relatively good agreement between the calculated and experimental values is seen (especially for higher modes). This can be explained by the inhomogeneous stress

Fig. $S(5)$ shows the frequency dependences of the piezoelectric resonances measured across the membranes in five points. The measurements were done in order to match the resonance mode shapes [Fig. S4(b)] with the experimental results. Some of the resonance peaks are suppressed and some are amplified. This is because the measurement points (1-5) are located close to the nodes and antinodes of the vibrating membrane. Overall mode shapes shown in Table I are very close to the experimental ones, thus validating the used FEM approach.

As can be noticed from (1), further increase in the resonance frequency of the membranes could be achieved by decreasing their lateral dimensions, while keeping the tensile stress small enough to prevent breaking of the membranes.
Optical lithography extended its resolution capability down to $250 \mathrm{~nm}$, and using the ArF excimer laser (193 nm) could further decrease the size of the membranes. As it is well known, $\mathrm{X}$-ray lithography can be used to get the membrane size down to a few tens of nanometer. However, these structures are becoming difficult to fabricate due to the high aspect ratio. As such, the membranes with a thickness of several nanometers and $10 \mathrm{~nm}$ in size are currently feasible to combine with the graphene layer. This would extend the resonance frequency to the megahertz range. Control of the membrane stress is also essential in this case [20]. To fabricate thin $\mathrm{Si}_{3} \mathrm{~N}_{4}$ membranes with a thickness of less than $5 \mathrm{~nm}$ in a wafer, a new fabrication process that employs a polycrystalline-Si (poly-Si) sacrificial layer has been proposed and evaluated [21]. This fabrication process significantly minimizes damage to the membrane. Using this process, $\mathrm{Si}_{3} \mathrm{~N}_{4}$ membranes with the thicknesses of $3 \mathrm{~nm}$ were stably fabricated with small thickness variation.

One of the possible applications of the fabricated membranes is in the area of the DNA sequencing. In the 1990s, it was proposed that it might be possible to use nanopores as sensors for DNA [22]. If DNA could be transferred through a nanometer-sized nanopore in a linear fashion, this might serve as a device to read out the sequence of DNA in an ultrafast way, which is of obvious interest for genomics applications. Current readout of the nanoporous membranes is based on the measurements of ionic conductivity [23], while piezoelectric readout could be much faster and reliable. If sequencing with nanoporous membranes covered by graphene turns out to be feasible, the impact will be huge because it will remove the need for chemical modification, amplification, and surface adsorption of the DNA. Single-molecule DNA sequencing could be one of the promising directions. Further investigations are needed in this field.

On the other hand, membranes with lower resonance frequency might be required for biomedical applications, for example, for vibrational energy harvesting [24]. This could 
be achieved by thinning the membranes while increasing their size. However, this could turn their low tensile stress into compressive one and cause buckling [20]. Obviously, more efforts are needed in this direction.

\section{CONCLUSION}

In this article, we prepared piezoelectric membranes consisting of the oxidized amorphous $\mathrm{Si}_{3} \mathrm{~N}_{4}$ membranes sandwiched between a few layer graphene and $\mathrm{Al}$ electrodes. Such membranes exhibit clear piezoelectric resonances that were rationalized by the FEM calculations. The highest displacement in the center of a square membrane of $6.7 \times 6.7 \mathrm{~mm}^{2}$ was about $14 \mathrm{~nm} / \mathrm{V}$ and could be further enhanced by adjusting the geometry of the device. Such a displacement is comparable with that exhibited by the polymer membranes based on PVDF-TrFE and could be used in flexible/stretchable electronics, for example, in microfluidic devices or electric energy harvesting. Furthermore, such membranes should be sensitive to the mechanical stress and can be used as biocompatible sensors or future devices for DNA sequencing.

\section{REFERENCES}

[1] M. E. Lines and A. M. Glass, Principles and Applications of Ferroelectrics and Related Materials. Oxford, U.K.: Oxford Univ. Press, 2001

[2] A. L. Kholkin, D. A. Kiselev, L. A. Kholkine, and A. Safari, "Smart ferroelectric ceramics for transducer applications," in Smart Materials, 1st ed. Boca Raton, FL, USA: CRC Press, 2008, ch. 9, pp. 9.1-9.12.

[3] H. Takasu, "Ferroelectric memories and their applications," Microelectron. Eng., vol. 59, no. 1, pp. 237-246, Nov. 2001, doi: 10.1016/S01679317(01)00630-X.

[4] K.-A.-N. Duerloo, M. T. Ong, and E. J. Reed, "Intrinsic piezoelectricity in two-dimensional materials," J. Phys. Chem. Lett., vol. 3, no. 19, pp. 2871-2876, Sep. 2012, doi: 10.1021/jz3012436.

[5] M. T. Ong and E. J. Reed, "Engineered piezoelectricity in graphene," ACS Nano, vol. 6, no. 2, pp. 1387-1394, Feb. 2012, doi: $10.1021 / \mathrm{nn} 204198 \mathrm{~g}$.

[6] W. Wu, "Stretchable electronics: Functional materials, fabrication strategies and applications," Sci. Technol. Adv. Mater, vol. 20, no. 1, pp. 187-224, Mar. 2019, doi: 10.1080/14686996.2018.1549460.

[7] A. K. Geim and K. S. Novoselov, "The rise of graphene," Nature Mater., vol. 6, no. 3, pp. 183-191, Mar. 2007, doi: 10.1038/nmat1849.

[8] K. E. El-Kelany, P. Carbonnière, A. Erba, J.-M. Sotiropoulos, and M. Rérat, "Piezoelectricity of functionalized graphene: A quantummechanical rationalization," J. Phys. Chem. C, vol. 120, no. 14, pp. 7795-7803, Mar. 2016, doi: 10.1021/acs.jpcc.5b11929.

[9] R. Skomski, P. A. Dowben, M. S. Driver, and J. A. Kelber, "Sublattice-induced symmetry breaking and band-gap formation in graphene," Mater. Horiz., vol. 1, no. 6, pp. 563-571, Aug. 2014, doi: $10.1039 / \mathrm{c} 4 \mathrm{mh} 00124 \mathrm{a}$

[10] M. T. Ong, K.-A.-N. Duerloo, and E. J. Reed, "The effect of hydrogen and fluorine coadsorption on the piezoelectric properties of graphene," J. Phys. Chem. C, vol. 117, no. 7, pp. 3615-3620, Feb. 2013, doi: 10.1021/jp3112759.

[11] S. Chandratre and P. Sharma, "Coaxing graphene to be piezoelectric," Appl. Phys. Lett., vol. 100, no. 2, Jan. 2012, Art. no. 023114, doi: 10.1063/1.3676084

[12] G. da Cunha Rodrigues, P. Zelenovskiy, K. Romanyuk, S. Luchkin, Y. Kopelevich, and A. Kholkin, "Strong piezoelectricity in single-layer graphene deposited on $\mathrm{SiO}_{2}$ grating substrates," Nature Commun., vol. 6, no. 1 , Nov. 2015, Art. no. 7572, doi: 10.1038/ncomms8572

[13] E. A. Irene, N. J. Chou, D. W. Dong, and E. Tierney, "On the nature of CVD Si-rich $\mathrm{SiO}_{2}$ and $\mathrm{Si}_{3} \mathrm{~N}_{4}$ films," J. Electrochem. Soc., vol. 127, no. 11, pp. 2518-2521, May 1980, doi: 10.1149/1.2129507.

[14] A. D. Ushakov, N. Yavo, E. Mishuk, I. Lubomirsky, V. Y. Shur, and A. L. Kholkin, "Electromechanical measurements of Gd-doped ceria thin films by laser interferometry," KnE Mater. Sci., vol. 1, pp. 177-182, Oct. 2016, doi: 10.18502/kms.v1i1.582.
[15] A. L. Kholkin, C. Wütchrich, D. V. Taylor, and N. Setter, "Interferometric measurements of electric field-induced displacements in piezoelectric thin films," Rev. Sci. Instrum., vol. 67, no. 5, pp. 1935-1941, May 1996, doi: $10.1063 / 1.1147000$.

[16] C. Z. Tan and J. Arndt, "Measurement of piezoelectricity in quartz and electrostriction in $\mathrm{SiO}_{2}$-glass by interferometric method," Phys. B, Condens. Matter, vol. 225, no. 3, pp. 202-206, Jul. 1996, doi: 10.1016/09214526(96)00284-0

[17] Y. A. Yildirim, A. Toprak, and O. Tigli, "Piezoelectric membrane actuators for micropump applications using PVDF-TrFE," J. Microelectromech. Syst., vol. 27, no. 1, pp. 86-94, Feb. 2018, doi: 10.1109/JMEMS.2017.2773090.

[18] N. H. Fletcher, Acoustic Systems in Biology. New York, NY, USA Oxford Univ. Press, 1992.

[19] M. Mikolajūnas, D. Viržonis, V. Grigaliūnas, S. Tamulevičius, and R. Kaliasas, "An impact of the residual stress on the sacrificial release of microelectromechanical membranes," Mater. Sci., vol. 13, no. 1, pp. 14-17, Apr. 2007.

[20] G. Abadias et al., "Review article: Stress in thin films and coatings: Current status, challenges, and prospects," J. Vac. Sci. Technol. A, Vac. Surf. Films, vol. 36, no. 2, Mar. 2018, Art. no. 020801, doi: 10.1116/1.5011790

[21] I. Yanagi, T. Ishida, K. Fujisaki, and K.-I. Takeda, "Fabrication of 3-nmthick $\mathrm{Si}_{3} \mathrm{~N}_{4}$ membranes for solid-state nanopores using the poly-Si sacrificial layer process," Sci. Rep., vol. 5, no. 1, Oct. 2015, Art. no. 14656, doi: 10.1038/srep14656.

[22] D. W. Deamer and M. Akeson, "Nanopores and nucleic acids: Prospects for ultrarapid sequencing," Trends Biotechnol., vol. 18, no. 4, pp. 147-151, Apr. 2000, doi: 10.1016/s0167-7799(00)01426-8.

[23] C. Dekker, "Solid-state nanopores," Nature Nanotechnol., vol. 2, no. 4, pp. 209-215, Mar. 2007, doi: 10.1038/nnano.2007.27.

[24] L. Dong, M. Grissom, and F. T. Fisher, "Resonant frequency of massloaded membranes for vibration energy harvesting applications," Energy, vol. 3, no. 3, pp. 344-359, Aug. 2015, doi: 10.3934/energy.2015.3.344.

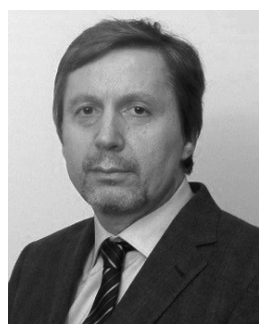

Andrei L. Kholkin (Fellow, IEEE) received the Ph.D. degree from the A. F. loffe PhysicalTechnical Institute, St. Petersburg, Russia, in 1987.

He held research positions at IFW, Dresden, Germany, EPFL, Lausanne, Switzerland, and Rutgers University, New Brunswick, NJ, USA. $\mathrm{He}$ is a Research Coordinator and the Head of the Laboratory of Advanced Microscopy of Functional Materials, University of Aveiro, Aveiro, Portugal. His laboratory develops multifunctional materials (ferroelectrics, multiferroics, piezoelectrics, energy materials, and graphene) and scanning probe microscopy techniques for their study. $\mathrm{He}$ has coauthored over 500 technical articles in this area including numerous reviews and book chapters.

Dr. Kholkin is a member of the IEEE Materials Research Society, and Portuguese Materials Society. He has been a Chair and Technical Committee Member of several international conferences and cofounded a new conference series on Piezoresponse Force Microscopy. He was a Guest Editor of the Special Issues on ferroelectrics in the IEEE TRANSACTIONS ON ULTRASONICS, FERROELECTRICS, AND FREQUENCY CONTROL and the Journal of Applied Physics and Materials Research Society Bulletin. In 2015, he won a prestigious IEEE Ferroelectrics Recognition Award for his studies on the nanoscale properties of ferroelectrics and piezoelectrics.

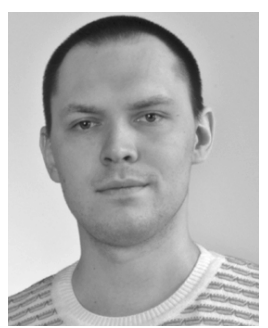

Andrei D. Ushakov (Member, IEEE) received the master's degree in nanotechnology and microsystems from the Institute of Natural Sciences and Mathematics, Ural Federal University, Ekaterinburg, Russia, in 2015, where he is currently working toward the Ph.D. thesis regarding the domain structure of multiaxial ferroelectrics.

$\mathrm{He}$ is a Junior Researcher with the Department of Optoelectronics and Semiconductor Technology, Ural Federal University. He is a coauthor of 17 research articles. His research interests include ferroelectrics, their domain structure, laser interferometry, and MEMS. 


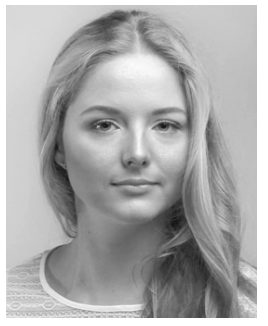

Maria A. Chuvakova is currently pursuing the Ph.D. degree with the Institute of Natural Sciences and Mathematics, Ural Federal University, Ekaterinburg, Russia.

She is a Junior Researcher with Ferroelectric Laboratory, Department of Optoelectronics and Semiconductor Technology. She has authored or coauthored 15 articles and more than 80 abstracts. Her current research interests include ferroelectric materials and their domain structure, nonlinear optical devices based on ferroelectrics, and photolithography.

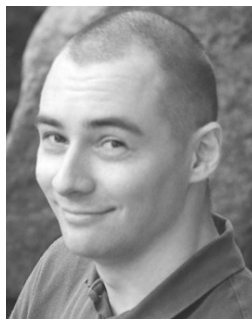

Mikhail S. Kosobokov received the Ph.D. degree in solid-state physics from Ural Federal University, Ekaterinburg, Russia, in 2016.

$\mathrm{He}$ is a Certified Specialist in the finiteelement analysis by COMSOL. He is currently a Research Staff Member of the Ferroelectric Laboratory, Ural Federal University. $\mathrm{He}$ is a coauthor of 12 research articles. His research interests include ferroelectrics materials, photonic crystals, microfluidics, laser action on the surface of solids, and FEM analysis of the multiphysics processes.

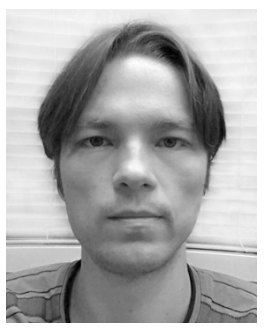

Andrey R. Akhmatkhanov was born in Sverdlovsk, Russia, on April 19, 1986. He received the M.S. and Ph.D. degrees in physics from Ural State University, Ekaterinburg, Russia, in 2008 and 2012, respectively.

In 2005, he joined Ferroelectrics Laboratory, Institute of Natural Sciences and Mathematics, Ural Federal University. He is currently engaged in research of domain structure evolution in uniaxial ferroelectrics and development of periodical poling techniques in these materials. $\mathrm{He}$ is a coauthor of 60 articles in peer-reviewed journals.

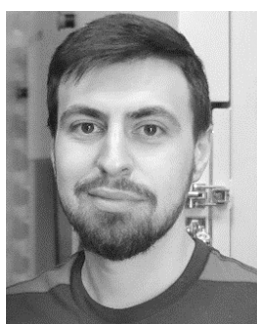

Andrei V. Turutin received the master's degree in electronics and nanoelectronics from the National University of Science and Technology MISiS (NUST MISiS), Moscow, Russia, in 2016, where he is currently pursuing the Ph.D. degree. His Ph.D. work includes research in composite magnetoelectric materials under the supervision of Prof. Dr. S. P. Kobeleva (NUST MISiS) and Prof. Dr. N. A. Sobolev (University of Aveiro, Aveiro, Portugal).

He has coauthored 16 research articles in peer-reviewed journals. His research interests include magnetoelectric effect in multiferroics, vibration sensors, and energy harvesters based on piezoelectrics.

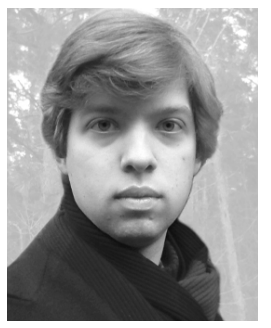

Maxim V. Chichkov received the master's degree in automatization from the National University of Science and Technology MISiS (NUST MISiS), Moscow, Russia, in 2015, where he is currently pursuing the Ph.D. degree with the Laboratory of Functional LowDimensional Structures, under the supervision of Prof. Dr Yu. N. Parkhomenko (NUST MISiS) and Dr. S. V. Morozov (IMT RAS) in collaboration with the Institute of Microelectronics Technology and High Purity Materials RAS, Chernogolovka,

Moscow, Russia.

His research microelectronics.

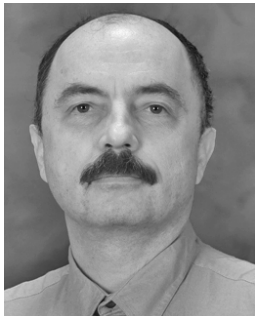

Ivan I. Kravchenko received the M.S. degree in materials sciences from Kyiv State University, Kyiv, Ukraine, in 1982, and the Ph.D. degree in solid-state physics from the Institute of Metal Physics, Ukrainian National Academy of Sciences, in 1993. He received Post-Doctoral Training with the University of Wisconsin-Madison, Madison, WI, USA.

$\mathrm{He}$ is currently a Research and Development Staff Member with Center for Nanophase Materials Sciences (CNMS), Oak Ridge National Laboratory, Oak Ridge, TN, USA. His general research interests include nanotechnology, particularly development and manufacture of metamaterials and novel optics elements. Other areas of exploration lay in development and manufacture of electronic components based on wideband semiconductors, layered as well as 1-D materials.

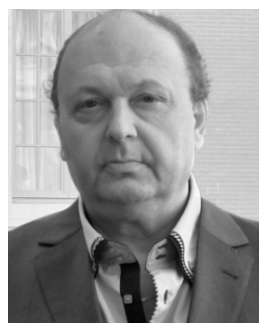

Yakov Kopelevich is currently a Full Professor in physics at the State University of Campinas (UNICAMP), Campinas, Brazil. He is an expert in physics of superconductivity, magnetism, carbon-based materials, semimetals, strongly correlated systems, and ferroelectricity. $\mathrm{He}$ is the author of more than 100 research articles published in leading scientific journals including Science, Nature, Physical Review Letters, Advanced Materials. He holds nine U.S. patents. His works were cited $\sim 3800$ times.

Dr. Kopelevich is a recipient of the university prize for outstanding achievements in teaching and science (University of Campinas, Brazil), Mercator Professorship at the University of Leipzig (Germany), the Lady Davis Professorship Award at the Hebrew University of Jerusalem (Israel), and Professorship at the University of Aveiro (Portugal) within the Erasmus Mundus, Joint European Masters in Materials Science Program. He was a Research Fellow at the Hewlett-Packard Laboratories (Palo Alto, CA, USA), University of Montpellier (CNRS, France), and LNMCI (CNRS, Toulouse, France). Seven Ph.D. and three master's theses were defended under his supervision. He was a PI of various research projects that received $\sim$ US\$ $6 \mathrm{M}$, and a Chair or member of program and organizing committees of various international conferences and he has been a member of the Advising Committee at the National Council for Scientific and Technological Development (CNPq) of Brazil.

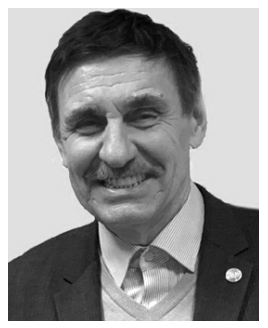

Vladimir Ya. Shur (Senior Member, IEEE) was born in Sverdlovsk, Russia, on September 15, 1945. He received the M.S. degree in physics, and the Ph.D. and Dr. Phys.-Math. Sci. degrees from the Ural State University, Ekaterinburg, Russia, in 1967, 1974, and 1990, respectively.

He founded the Ferroelectric Laboratory in the same University in 1982 . He is currently engaged in research on the kinetics and statics of the domain structure in ferroelectrics, and micro- and nanodomain engineering in lithium niobate and lithium tantalate for nonlinear optical applications. He is an author or coauthor of more than 230 publications in peer-reviewed journals and five book chapters. 